\title{
PAPER
}

\section{Immediate and delayed effects of risperidone on cerebral metabolism in neuroleptic naïve schizophrenic patients: correlations with symptom change}

\author{
E T C Ngan, C J Lane, T J Ruth, P F Liddle
}

J Neurol Neurosurg Psychiatry 2002;72:106-110

See end of article for authors' affiliations

....... a.

Correspondence to: Dr E Ngan, Department of Psychiatry, University of British Columbia, 2255 Wesbrook Mall, Vancouver, BC, Canada V6T 2Al;

ngan@interchange.ubc.ca

Received 14 March 2001 In revised form 14 August 2001

Accepted 24 August 2001
Objective: Different symptom patterns have been shown to be associated with specific patterns of cerebral metabolic activity in schizophrenia. Treatment with various neuroleptic drugs results in decreased metabolism in frontal cortical regions. The temporal and regional relation between changes in metabolism and symptom improvement after treatment with risperidone was studied in eight previously unmedicated schizophrenic patients.

Method: Cerebral metabolic activity was measured using PET before neuroleptic exposure, after the first dose of risperidone, and after 6 weeks of treatment. Pearson correlations were calculated for regions of significant change in metabolism and symptom change.

Results: After 6 weeks of treatment significant deactivations were seen in the left lateral cortical frontal region and medial frontal cortex. Significant changes were detectable in the medial frontal region 90 minutes after the first dose of risperidone. Patients with higher baseline activity in the identified medial frontal cluster had higher baseline positive symptom scores and reduction in medial frontal metabolism was correlated with reduction in positive symptom score.

Conclusion: The evidence suggests that the reduction in medial-frontal activity after treatment with risperidone is a direct effect of risperidone and not a consequence of symptom improvement. Reduction of medial frontal metabolism may be one of the physiological mechanisms by which risperidone alleviates symptoms of psychosis in schizophrenia.
M ost studies that have examined the pattern of cerebral activity associated with psychotic symptoms suggest that positive psychotic symptoms, such as delusions, hallucinations, and disorganisation phenomena are associated with overactivity of the frontal and temporal cortex, thalamus, and basal ganglia. For example, disorganisation is associated with overactivity of the medial frontal $\operatorname{cortex}^{1-3}$ and thalamus. ${ }^{1}$ Delusions and/or hallucinations have been reported to be associated with overactivity in the temporal lobe, ${ }^{145}$ frontal cortex $^{56}$ and ventral striatum. ${ }^{15}$ However, there are some discrepancies in the data, Ebmeier et al found that delusions and hallucinations were associated with temporal lobe underactivity. ${ }^{2}$ Andreasen et al found that acute schizophrenia was associated with frontal underactivity. ${ }^{7}$ Overall, most of the evidence supports the hypothesis that delusions, hallucinations, and disorganisation phenomena are associated with cortical overactivity, and also overactivity in at least some subcortical regions, particularly the ventral striatum.

The anatomical location and the time course of the changes in cerebral function associated with the therapeutic effects of antipsychotic drugs remain a subject of debate. Previous studies indicate that antipsychotic treatment causes a decrease in cortical metabolism, especially frontal metabolism ${ }^{8-11}$ and an increase in subcortical metabolism. ${ }^{8}{ }^{12-15}$ Several studies have reported that sensitivity to change in regional metabolism after administration of antipsychotic drugs is correlated with treatment responsiveness. ${ }^{8}{ }^{15} 16$

Taken together, the findings of the effect of antipsychotic drugs on cerebral metabolism and the patterns of cerebral activity associated with psychotic symptoms suggest that the mechanism of antipsychotic action should produce a reduction in cortical metabolism, especially in the frontal and temporal cortex.
Although placebo controlled trials indicate that the antipsychotic effect of neuroleptic medications become statistically significant over a period of several weeks ${ }^{17}$ the reduction in psychotic symptoms is usually non-significantly greater in patients receiving the active drug from the first assessment time point. ${ }^{18}$ Furthermore, clinical observation indicates that the effects on mental state can be discerned within hours of the first dose. The immediate effects include not only a reduction in agitation (which might be attributed to any of several pharmacological mechanisms) but also extrapyramidal effects such as acute dystonia, which are likely to arise from blockade of dopamine receptors. ${ }^{19}$ Studies with PET demonstrate that D2 receptor occupancy can reach $80 \%$ within 2 hours of administration of a single dose of risperidone $(4 \mathrm{mg}) .^{20}$ This suggests the possibility that the therapeutic effect may evolve gradually from the beginning of treatment. The failure to detect significant improvement in the early phase of treatment may be due to lack of statistical power to detect relatively small effects. The question of whether the immediate effects of antipsychotic medication on cerebral function evolve and are related to subsequent therapeutic effects might in principle be addressed by a longitudinal study employing PET to measure regional cerebral glucose metabolism. Furthermore, a longitudinal study offers the possibility of determining whether or not a specific cerebral change precedes symptom change, or vice versa. Such information would help determine whether the relevant cerebral metabolic change is related to the cause of symptom reduction, or merely the consequence of it.

Abbreviations: $F D G$, fluorodeoxy glucose; $\mathrm{CGl}_{1}$, clinical global impression severity scale; SANS, scale for assessment of negative symptoms; SAPS, scale for assessment of positive symptoms; EPS, extrapyramidal side effects 
In this study we investigated the effects of risperidone on cerebral metabolism 90 minutes after first exposure to risperidone and after 6 weeks of treatment in a group of first episode schizophrenic patients. We studied first episode patients to avoid possible confounding effects of prior antipsychotic treatment, and also because a substantial reduction in symptom severity might be expected after 6 weeks of treatment with moderate doses of antipsychotic medication in a substantial proportion of such patients. We employed risperidone because low to moderate doses of this antipsychotic drug produce relatively few extrapyramidal side effects, which might be associated with changes in metabolism that would confound the interpretation of the results. The timing of the second scan was selected so as to detect change that occurred very soon after the administered drug entered the brain, and before any adaptive changes mediated by gene expression would be anticipated. Ideally, measurement at additional time points would be more informative; three scans a patient is the maximum that is feasible using PET with the ligand fluorodeoxy glucose (FDG), on account of radiation exposure.

We tested the hypothesis that 6 weeks of treatment with risperidone would lead to a decrease in activity in frontal and temporal cortical regions. We explored the relation between changes found and clinical improvement. For areas where there is a significant relation between metabolic changes after 6 weeks of treatment and symptom improvement we performed a directed search for significant changes after the first dose of risperidone.

\section{METHOD}

Eight patients meeting DSM IV ${ }^{21}$ criteria for schizophrenia or schizophreniform psychosis with no history of exposure to neuroleptic drugs were recruited into this study. They each gave written informed consent for their participation. Illness severity was rated on the clinical global impression severity scale $\left(\mathrm{CGI}_{1}\right.$; a seven point scale on which $\mathrm{l}=$ normal, $2=$ borderline ill, $3=$ mildly ill, $4=$ moderately ill, $5=$ markedly ill, $6=$ severely ill, and $7=$ among the most extremely ill patients). Clinical improvement at 6 weeks was rated on the clinical global impression improvement scale (CGI ; a seven point scale on which $1=$ very much improved, $2=$ much improved, $3=$ minimally improved, $4=$ no change, $5=$ minimally worse, $6=$ much worse, and $7=$ very much worse). Symptom severity was assessed using the scale for assessment of negative symptoms (SANS) and scale for assessment of positive symptoms (SAPS) ${ }^{22}$ at baseline and after 6 weeks of treatment. Benzodiazepine treatment was allowed for the management of anxiety and agitation before the first scan. Extrapyramidal side effects (EPS) were monitored using the Simpson Angus scale for parkinsonian symptoms. ${ }^{23}$

On the first scanning day, the patients underwent two PET scans, one after administration of a placebo tablet and the other after administration of risperidone, under single blind conditions. For each scan, 2 mc FDG were given by bolus injection delivered over 1 minute. A placebo tablet was administered 90 minutes before the injection of FDG for the first scan. Immediately after completion of the first scan, $2 \mathrm{mg}$ risperidone was administered orally, 90 minutes before the injection of FDG for the second scan. The patients were blind to the order of administration of risperidone and placebo.

For each scan, arterialised venous blood samples were taken over 120 minutes after the bolus injection to provide an estimate of blood activity curves for the residual correction method described below. To maintain a similar mental state during uptake of ${ }^{18} \mathrm{~F}$-fluorodeoxyglucose patients engaged in a continuous performance task in which they were instructed to press a response button whenever two consecutive letters presented sequentially on a computer monitor were identical. Scans were obtained commencing 40 minutes after each injection of FDG with an ECAT 953B PET Camera (Knoxville
TN, USA) with between plan septa retracted for three dimensional image acquisition. ${ }^{24}$ Data were collected in 31 contiguous slices covering a $10.8 \mathrm{~cm}$ field of view. Images were acquired in four consecutive 5 minute frames to allow for realignment due to intrascanner movement over the acquisition period. Correction for radiation absorption was made using data from a transmission scan employing a germanium ring source. Ten minutes before administration of the second injection of FDG, a "preinjection" scan of 10 minutes in duration was performed to measure residual activity from the first tracer injection.

Patients received $2 \mathrm{mg}$ risperidone on the second day and 4 mg (in divided dosages) on the third day. Dosages were adjusted to a maximum of $6 \mathrm{mg} /$ day as clinically indicated. Dosages were decreased as necessary to minimise side effects. After 6 weeks of treatment a third (post-treatment) scan was performed 90 minutes after the morning dose of risperidone.

\section{Data analysis}

Image analysis was performed using Statistical Parametric Mapping software (SPM96; Wellcome Department of Cognitive Neurology, London, UK). The four 5 minute frames from each scan session were realigned to the first frame and averaged. The residual activity from the first tracer injection at the time of the second scan acquisition was calculated using individual estimates of the rate coefficient for the loss of tracer from the metabolic pool due to dephosphorylation (k4) for each patient. The method uses the residual activity measured before the second injection and the measured plasma activity curve fitted to a four exponential function to derive individual estimates of k4 using the Phelps-Sokoloff equations for glucose metabolism ${ }^{25}$ for each patient.

This procedure was tested in four patients who did not receive a second injection of FDG. The mean discrepancy in predicted residual activity and measured activity was less than $4 \%$ (range $1.5 \%-7.9 \%$ ).

The residual activity from the baseline scan was subtracted from the scan after the second dose to produce a "corrected second scan". This "corrected image" was used for all subsequent analysis. The averaged images for each of the three scan sessions were aligned to the image from the baseline session and a mean image produced from which normalisation parameters for spatial normalisation to the PET image template in SPM96 were determined. After normalisation images were smoothed with a $10 \mathrm{~mm}$ gaussian filter resulting in final images with smoothness of 13.3, 15.1, and $12.1 \mathrm{~mm}$ (full width half maximum) in the $\mathrm{x}, \mathrm{y}$, and $\mathrm{z}$ directions respectively.

To control for variation in global image intensity between patients normalised metabolic images were created by proportional scaling after ensuring that there were no significant differences in group mean global metabolism across the three conditions. Differences in normalised metabolism were calculated voxel by voxel with correction for multiple comparisons based on the theory of random gaussian fields employed in SPM 96 for both the first dose-baseline contrast and the post-treatment baseline contrast. When examining changes in individual voxels after 6 weeks of treatment, the primary criterion for statistical significance was $p<0.05$ after correcting for multiple comparisons assuming examination of the entire brain. In addition, we identified significant clusters of contiguous suprathreshold voxels, satisfying the criterion $\mathrm{p}<0.05$ after correcting for multiple comparisons, determined by the test for cluster significance proposed by Friston $e t a^{26}$ and implemented in SPM. The threshold for inclusion of a voxel in a cluster was $z=2.33$ (corresponding to $\mathrm{p}<0.01$ ). In the case of the changes after the first dose, the risk of type 2 error when applying a stringent test for multiple comparisons is especially high because the anticipated changes in metabolism are small. Therefore, when examining the changes after 


\begin{tabular}{|llll|}
\hline Table $1 \quad$ Patient demographics & & \\
\hline Demographic variable & Mean & SD \\
\hline Age (y) & 26.5 & 5.6 \\
GAS at admission & 31.9 & 6.5 \\
Lifetime stays in hospital (months) & 0.2 & 0.2 \\
Clinical global impression severity & 4.1 & 1.0 \\
Sex (female:male) & $6: 2$ & \\
Handedness (right:left) & $7: 1$ & \\
\hline
\end{tabular}

the first dose, we initially examined only selected voxels. Our main objective in studying the effects of a single dose was to determine whether or not change could be discerned after the first dose at the sites where significant change developed after 6 weeks. Therefore, we selected the voxel at which the change after 6 weeks was most significant, within each of the clusters that were significant after 6 weeks of treatment. We then examined the significance of change after the first dose at these selected voxels, applying a criterion of $p<0.01$, uncorrected.

We examined the relation between symptoms and normalised metabolism in the identified significant clusters. Pearson correlations between baseline metabolism, change in metabolism, baseline symptom severity, and change in symptom severity were calculated. Clinical variables used in the bivariate analysis included a positive symptom score (sum of global items in SAPS), negative symptom score (sum of global avolition, anhedonia, alogia, and affective flattening), a disorganisation score (positive formal thought disorder, inappropriate affect, and poverty of content) and a score for reality distortion (sum of hallucination global and delusions global scores). ${ }^{27}$ It should be noted that the items contributing to a global score for positive symptoms overlap with those contributing to scores for reality distortion and disorganisation. We examined the positive/negative dichotomy because many treatment trials examine therapeutic effects on these two groups of symptoms. We also examined the reality distortion/ disorganisation/psychomotor poverty trichotomy because of evidence that these three clusters of symptoms might be associated with different patterns of brain activity. ${ }^{1}$

\section{RESULTS}

\section{Clinical effects}

Patient demographics are shown in table 1. One patient was considered severely ill, one markedly ill, four moderately ill, and 2 mildly ill $\left(\mathrm{CGI}_{1}\right)$ at baseline. Four patients were very much improved, three patients much improved, and one patient minimally improved $\left(\mathrm{CGI}_{2}\right)$ after 6 weeks of treatment. The mean improvement at 6 weeks in positive symptoms was $78.1 \%$ (range $50 \%-100 \%$ ), negative symptoms $53.0 \%$ (range $20 \%-100 \%$ ), disorganisation $68.7 \%$ (range $40 \%-100 \%$ ) and reality distortion $75.7 \%$ (range $0 \%-100 \%$ ). The reduction in positive symptom severity was significantly correlated with baseline positive symptom severity $(r=0.92, \mathrm{p}=0.001, \mathrm{df}=7)$. Two patients had their dosage of risperidone reduced during the course of treatment due to emergent extrapyramidal symptoms (EPS). These two patients had discernable EPS at 6 weeks. The remaining six patients showed no detectable EPS.

\section{Changes in metabolism after 6 weeks of treatment}

There were two extensive clusters of voxels in which there were decreases in metabolism after 6 weeks (fig 1). The largest cluster, located predominantly in the left lateral frontal cortex, embracing both middle and inferior frontal gyri, comprised $5400(2 \times 2 \times 2 \mathrm{~mm})$ suprathreshold voxels. The significance of the cluster as a whole was $\mathrm{p}<0.001$, corrected for multiple comparisons. The mean change in activity in this cluster after 6 weeks of treatment was $-7.8 \%$. The most significant reduction in an individual voxel was located at $\mathrm{x}, \mathrm{y}$, $\mathrm{z}=-10,34,-18 \mathrm{~mm}(\mathrm{z}=4.96 ; \mathrm{p}=0.008$ corrected for multiple comparisons).

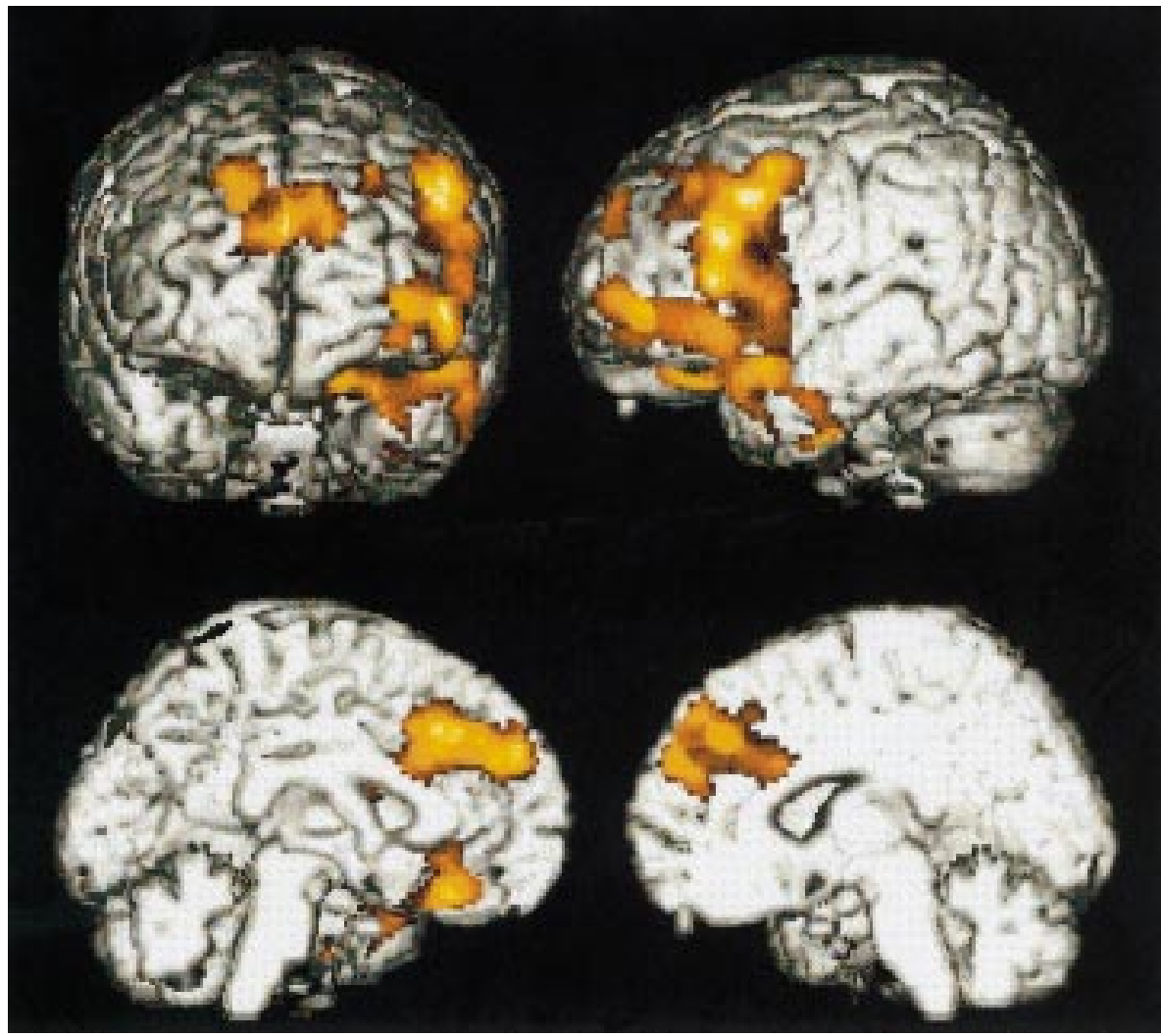

Figure 1 Surface rendering of areas of significant reduction in normalised metabolism after 6 weeks of treatment with risperidone. 
The second largest cluster was located in medial frontal cortex. This cluster embraced 2456 suprathreshold voxels. The significance of the cluster as a whole was $p=0.004$, corrected for multiple comparisons. The mean change in activity in this cluster after 6 weeks of treatment was $-7.6 \%$. The most significant reduction in an individual voxel was located at $\mathrm{x}, \mathrm{y}$, $\mathrm{z}=-4,24,32 \mathrm{~mm}(\mathrm{z}=4.56 ; \mathrm{p}=0.04$ corrected for multiple comparisons)

There was a small extension of the left lateral cluster into the left temporal lobe. The most significant reduction in metabolism in an individual voxel in the left temporal lobe cluster was located at $\mathrm{x}, \mathrm{y}, \mathrm{z}=-34,-6,-40 \mathrm{~mm} \quad(z=4.22$; $\mathrm{p}<0.0001$ uncorrected; $\mathrm{p}=0.146$ corrected for multiple comparisons).

There were no changes in metabolism in the basal ganglia that remained significant after correcting for multiple comparisons. In the ventral striatum, there was a small cluster of voxels in which metabolism decreased after treatment (peak significance at $\mathrm{x}, \mathrm{y}, \mathrm{z}=6,8,-8 \mathrm{~mm} ; \mathrm{z}=3.22, \mathrm{p}=0.0006$, uncorrected).

\section{Changes in metabolism after the first dose}

For the most significant voxel after 6 weeks of treatment in the large suprathreshold cluster extending from the lateral frontal cortex to the orbital frontal cortex, there was no evidence of a significant change after the first dose. In the cluster in the medial frontal cortex, for the voxel exhibiting the most significant reduction in metabolism after 6 weeks treatment (located at $\mathrm{x}, \mathrm{y}, \mathrm{z}=-4,24,32 \mathrm{~mm}$ ), there was a significant reduction after the first dose $(z=2.54 ; \mathrm{p}=0.006$, uncorrected $)$. This voxel was part of a cluster of 117 suprathreshold voxels. The most significant voxel in this cluster was located at $x, y$, $z=-6,30,40 \quad(z=3.64, p=0.0001$, uncorrected $)$. The mean change in activity in this cluster after a single dose of risperidone was $-5.8 \%$.

In an undirected search of the entire brain, there were no changes in metabolism after the first dose that were significant after a stringent correction for multiple comparisons. In view of the risk of type 2 errors when applying a stringent criterion for significance, we note that there were changes in the lateral frontal cortex and temporal lobe that satisfied less stringent criteria. In view of the prior evidence implicating the lateral frontal cortex and temporal lobes in the expression of schizophrenic symptoms ${ }^{156}$ less stringent criteria might be justified. None the less, these findings should be interpreted with caution. In the lateral frontal cortex, the most significant change was located at $x, y, z=-36,56,8(z=3.25$, $\mathrm{p}=0.0006$, uncorrected) within a contiguous cluster of 310 suprathreshold voxels. In the temporal cortex, the most significant change was at $x, y, z=-50,-16,-8, \quad(z=3.32$, $\mathrm{p}=0.0004$, uncorrected) within a contiguous cluster of 71 suprathreshold voxels.

\section{Correlations between symptoms and metabolism}

The baseline metabolism in the significant cluster of 2456 voxels in medial frontal cortex in which metabolism subsequently decreased during 6 weeks of treatment was positively correlated with baseline positive symptoms $(r=0.80$, $\mathrm{p}=0.016)$ and subsequent change in positive symptoms $(r=0.85, \mathrm{p}=0.008)$ and change in disorganisation $(r=0.84$, $\mathrm{p}=0.009$, table 2 ).

The change in activity in this cluster after 6 weeks of treatment was positively correlated with baseline positive symptoms $(r=0.75, \mathrm{p}=0.031)$ and with change in positive symptoms $(r=0.74, \mathrm{p}=0.037)$. There were no significant correlations between baseline or change in activity in the left lateral frontal cluster and any baseline or symptom change measures used in this analysis. There were no significant correlations between baseline negative symptom severity, improvement in negative symptoms, and measures in metabolism.
Table 2 Correlations of baseline symptom severity and change in symptom severity after 6 weeks treatment, with baseline metabolism and change in metabolism after 6 weeks treatment in the significant cluster of 2456 voxels in the medial frontal cortex, in which metabolism subsequently decreased during 6 weeks treatment

\begin{tabular}{|c|c|c|}
\hline & $\begin{array}{l}\text { Baseline } \\
\text { metabolism }\end{array}$ & $\begin{array}{l}\text { Change in } \\
\text { metabolism }\end{array}$ \\
\hline \multicolumn{3}{|l|}{ Baseline symptom severity: } \\
\hline Disorganization & 0.35 & 0.04 \\
\hline Reality distortion & 0.27 & 0.38 \\
\hline Total positive symptoms & $0.80^{*}$ & $0.75^{*}$ \\
\hline \multicolumn{3}{|l|}{ Change in symptom severity: } \\
\hline Disorganisation & $0.84^{* *}$ & 0.54 \\
\hline Reality distortion & 0.42 & 0.39 \\
\hline Total positive symptoms & $0.85^{* *}$ & $0.74^{*}$ \\
\hline
\end{tabular}

\section{DISCUSSION}

Risperidone produces decreases in metabolism in the frontal cortex, consistent with previous findings. ${ }^{10}$ This reduction was found in both the medial and the lateral frontal cortex. In accord with the prediction based on prior findings that medial prefrontal overactivity is associated with severity of disorganisation ${ }^{1-3}$ and with hallucinations, ${ }^{5}$ we found that the decrease in medial frontal cortex metabolism is related to alleviation of positive symptoms. This association can be accounted for largely by the finding that in this region, baseline metabolism is correlated with baseline severity of symptoms.

There was evidence for discernible changes in both medial and left lateral frontal cortex within 2 hours of the first dose, although these reductions were less extensive than those found after 6 weeks of treatment. The fact that a decrease in metabolism in the medial frontal cortex is discernible within 2 hours of the first dose, at a time when the degree of symptom resolution would be expected to be minimal, suggests that the decrease in metabolism is not merely a consequence of alleviation of symptoms.

Studies of cognitive activation with PET have fairly consistently reported diminished activation of the frontal lobe during a demanding relative to a less demanding baseline condition in schizophrenic patients. In particular, Spence et $a l^{28}$ found that schizophrenic patients exhibited recovery of the normal increase in activation in dorsolateral prefrontal cortex during a demanding motor task relative to a resting baseline, as symptoms resolved during treatment. Such a recovery could arise from increase in activity during the demanding task; a decrease during the non-demanding baseline condition; or both. In an fMRI study of first episode schizophrenic patients, Mendrek et al found a partial recovery of normal frontal activation during a demanding 2-back working memory task relative to that during a non-demanding 0-back condition, during treatment. ${ }^{29}$ Furthermore, Mendrek et al demonstrated that this recovery was largely due to a decrease in an abnormally high level of activity during the non-demanding 0-back condition, during treatment. In our study, patients performed a non-demanding continuous performance task that could also be described as a l-back working memory task, during uptake of the tracer. Our findings indicate that frontal activation during this relatively non-demanding condition decreases during treatment. They do not rule out the possibility that activity during a demanding task might increase relative to that during a non-demanding baseline condition.

In the absence of a healthy control group, it is not possible to ascertain whether or not the changes in metabolism are 
unique to patients. Furthermore, randomisation of the order of the first and second scans was not feasible in a study of patients, because such randomisation would have necessitated a substantial time interval between the scans, leading to a delay in initiation of treatment. Therefore it is not possible to exclude the possibility that the changes reflect scan-order effects, such as changes associated with a reduction in anxiety, which are unrelated to the pharmacological effect of risperidone treatment. However, in a separate study of 12 healthy subjects, in which a similar PET procedure was employed to measure metabolism 90 minutes after placebo and 90 minutes after $2 \mathrm{mg}$ risperidone, administered in random order 2 weeks apart, we found reductions in metabolism after risperidone at similar sites in the medial and lateral frontal cortex (Lane et al, unpublished data). These findings indicate that the reductions in metabolism at these sites are not entirely due to scan-order effects unrelated to treatment. Furthermore, the finding of a change in the medial frontal cortex in healthy subjects similar to that found to be associated with the subsequent reduction in severity of disorganisation in schizophrenic patients suggests that a part of the therapeutic effect of risperidone arises from a pharmacological effect that occurs in both patients and healthy subjects.

Our study does not have sufficient power to rule out the possibility of clinically relevant change in the temporal lobes or basal ganglia. We did not find a reduction in metabolism in the temporal lobe satisfying stringent criteria for significance. There was a small cluster of voxels in the left temporal lobe in which a suprathreshold decrease in metabolism occurred, but this cluster was not significant after correcting for multiple comparisons. However, the changes in the left temporal lobe might be regarded as significant if the object of the analysis had been to test a specific hypothesis regarding localised change in the temporal lobe, such as that reported by Liddle et $a l^{30}$ using this same data set. The sample size of eight patients increases the chances of both type 1 and type 2 errors and limits the generalisability of the results to the population at large.

\section{ACKNOWLEDGEMENTS}

This work was supported by the Norma Calder Foundation and Canadian Institute of Health Research

\section{Authors' affiliations}

E T C Ngan, C J Lane, P F Liddle, Department of Psychiatry, University of British Columbia, 2255 Wesbrook Mall, Vancouver, BC, Canada V6T 2Al

T J Ruth, TRIUMP Positron Emission Tomography program

\section{REFERENCES}

1 Liddle PF, Friston KJ, Frith D, et al. Patterns of cerebral blood flow in schizophrenia. Br J Psychiatry 1992;160:179-86.

2 Ebmeier KP, Blackwood DHR, Murray C, et al. Single photon emission tomography with $99 \mathrm{mTc}$-exametazime in unmedicated schizophrenic patients. Biol Psychiatry 1993:33:487-95.

3 Yuasa $S$, Kurachi $M$, Suzuki $M$, et al. Clinical symptoms and regional cerebral blood flow in schizophrenia. Eur Arch Psychiatry Clin Neurosci $1995 ; 246 ; 7-12$
4 Musalek M, Podreka I, Walter $\mathrm{H}$, et al.. Regional brain function in hallucinations: a study of regional cerebral blood flow with $99 \mathrm{~m}$-Tc-HMPAO-SPECT in patients with auditory hallucinations, tactile hallucinations, and normal controls. Comp Psychiatry 1989;30:99-108.

5 Silbersweig DA, Stern E, Frith CD. A functional neuroanatomy of hallucinations in schizophrenia. Nature 1995:378:176-9.

6 McGuire PK, Shah GMS, Murray RM. Increased blood flow in Broca's area during auditory hallucinations in schizophrenia. Lancet 1993;342:703-6.

7 Andreasen NC, O'Leary DS, Flaum M, et al. Hypofrontality in schizophrenia: distributed dysfunctional circuits in neuroleptic-naive patients. Lancet 1997:349:1730-4.

8 Potkin SG, Buchsbaum MS, Jin Y. Clozapine effects on glucose metabolic rate in striatum and frontal cortex. J Clin Psychiatry 1994;55(suppl B):63-6.

9 Holcomb HH, Cascella NG, Thaker GK, et al. Functional sites of neuroleptic drug action in the human brain: PET/FDG studies with and without haloperidol. Am J Psychiatry 1996;153:41-9.

10 Berman I, Merson A, Sison C. Regional cerebral blood flow changes associated with risperidone treatment in elderly schizophrenia patients: a pilot study. Psychopharmacology Bulletin 1996;32;95-100

11 Cohen RM, Nordahl TE, Semple WE, et al. The brain metabolic patterns of clozapine and fluphenazine treated patients with schizophrenia during a continuous performance task. Arch Gen Psychiatry 1997:54:481-6.

12 Buchsbaum MS, Wu J, De Lisi LE. Positron emission tomography studies of the basal ganglia and somatosensory cortex neuroleptic drug effects: differences between normal controls and schizophrenic patients. Biol Psychiatry 1987;22:479-94.

13 Buchsbaum MS, Potkin S, Siegel B. PET studies of drug interaction with brain regional glucose metabolism. Clin Neuropharmacol 1992;15:472-3

14 Buchsbaum MS, Potkin SG, Marshall JF. Effects of clozapine and thiothixene on glucose metabolic rate in schizophrenia. Neuropsychopharmacology 1992;6:155-63.

15 Buchsbaum MS, Potkin SG, Siegel BV. Striatal metabolic rate and clinical response to neuroleptics in schizophrenia. Arch Gen Psychiatry 1992:49:966-74.

16 Bartlett EJ, Brodie JD, Simkowitz P, et al. Effect of a haloperidol challenge on regional brain metabolism in neuroleptic-responsive and non-responsive schizophrenic patients. Am J Psychiatry 1998; 155:337-43.

17 Johnstone EC, Crow TJ, Frith CD, et al. Mechanism of the antipsychotic effect in the treatment of acute schizophrenia. Lancet 1978;i:848-51.

18 Beasley CM, Tollefson G, Tran P, et al. Olanzapine versus placebo and haloperidol: acute phase results of the North American double-blind olanzapine trial. Neuropsychopharmacology 1996;14:111-23

19 King DJ, Burke M, Lucas RA. Antipsychotic drug-induced dysphoria. Br J Psychiatry 1995;167:480-2.

20 Kapur S, Receptor occupancy by antipsychotics: concepts and findings. In: Lidow MS, ed. Neurotransmitter receptors in actions of antipsychotic medictions. Boca Rato: CRC Press, 2000:163-76.

21 American Psychiatric Association. Diagnostic and statistical manual of mental disorders, 4th ed. Washington, DC: American Psychiatric Press, 1994.

22 Andreasen NC, Arndt S, Alliger R, et al. Symptoms of schizophrenia: methods, meaning, and mechanism. Arch Gen Psychiatry 1995;52:341-51.

23 Simpson GM Angus JW. A rating scale for extrapyramidal side effects. Acta Psychiatr Scand (Suppl) 1970;212:11-19.

24 Sossi V, Oakes TR, Chan GL, et al. Quantitative comparison of three and two dimensional PETT with human brain studies. J Nucl Med 1998;39:1714-19.

25 Phelps ME, Huang SC, Hoffman EJ, et al. Tomographic measurement of local cerebral glucose metabolic rate in humans with (F-18) 2 fluoro-deoxy-D-glucose: validation of method. Ann Neurol 1979;6:371-88

26 Friston KJ, Holmes A, Poline JB, et al. Detecting activations in PET and fMRI: levels of inference and power. Neuroimage 1996;4:223-35.

27 Liddle PF. The symptoms of chronic schizophrenia: a re-examination of the positive-negative dichotomy. Br J Psychiatry 1987;151:124-51.

28 Spence SA, Hirsch SR, Brooks DJ, et al. Prefrontal cortex activity in people with schizophrenia and control subjects. Evidence from positron emission tomography for remission of "hypofrontality" with recovery from acute schizophrenia. Br J Psychiatry 1998;172:316-23.

29 Mendrek A, Laurens KR, Ngan E, et al. New insights into prefrontal function in schizophrenia. Schizophr Res 2001:49:181.

30 Liddle PF, Lane CJ, Ngan ETC. Immediate effects of risperidone on cortico-striato-thalamic loops and the hippocampus. BrJ Psychiatry 2000; 177:402-7. 